\title{
Dynamic Nomogram for Predicting Thrombocytopenia in Adults with Acute Pancreatitis
}

\author{
Hongbin Deng ${ }^{1, *}$ \\ Xianqiang $\mathrm{Yu}^{2, *}$ \\ Kun Gao' \\ Yang $\mathrm{Liu}^{3}$ \\ Zhihui Tong' \\ Yuxiu Liu (D) \\ Weiqin Li $\mathbb{D}^{1-3}$
}

'Department of Critical Care Medicine, Jinling Hospital, Nanjing Medical

University, Nanjing, People's Republic of China; ${ }^{2}$ School of Medicine, Southeast

University, Nanjing, People's Republic of China; ${ }^{3}$ Department of Critical Care

Medicine, Jinling Hospital, Medical School of Nanjing University, Nanjing, People's Republic of China

*These authors contributed equally to this work
Correspondence: Weiqin Li; Yuxiu Liu Email liweiqindr@nju.edu.cn; Liu_yuxiu@163.com
Objective: Thrombocytopenia increases the risk of hemorrhage in patients with acute pancreatitis (AP), leading to poor clinical outcomes. Currently, there is no reliable tool for the early assessment of thrombocytopenia in these patients. We aimed to develop a nomogram based on available clinical parameters and validate its efficacy in predicting thrombocytopenia.

Methods: This was a retrospective study. All the data were extracted from an electronic database from May 2018 to May 2019. Patients with a diagnosis of AP and staying in the intensive care unit for more than 3 days were retrospectively analyzed. A clinical signature was built based on reproducible features, using the least absolute shrinkage and selection operator method (LASSO), and logistic regression established the model $(\mathrm{P}<0.05)$. Nomogram performance was determined by its discrimination, calibration, and clinical usefulness.

Results: A total of 594 eligible patients were enrolled, of whom 399 were allocated to the training sets and the 195 in the test sets. The clinical features, including blood urea nitrogen (BUN), fibrinogen (FIB), and antithrombase III, were significantly associated with the incidence of thrombocytopenia after acute pancreatitis $(\mathrm{p}<0.05)$ in training sets. The individualized nomogram showed good discrimination in the training sample (area under the receiver operating characteristic curve [AUC], 0.881) and in the validation sample (AUC, 0.883 ) with good calibration.

Conclusion: The proposed nomogram has good performance for predicting thrombocytopenia in patients with acute pancreatitis and may facilitate clinical decision-making.

Keywords: acute pancreatitis, thrombocytopenia, systemic inflammatory response syndrome, coagulopathy, nomogram

\section{Introduction}

Acute pancreatitis (AP) is an inflammatory disease triggered by acinar cells with various clinical manifestations, and most patients can recover with conservative treatment. $^{1-4}$ The activation of inflammatory cells triggers the cascade release of a large number of inflammatory factors, leading to the disorder of pro-inflammatory and anti-inflammatory states, which can induce lethal severe acute pancreatitis (SAP) characterized by systemic inflammatory response syndrome (SIRS) and multiple organ dysfunction syndrome (MODS). ${ }^{5-9}$

The coagulopathy associated with SIRS is a manifestation of host immune dysfunction stimulated by severe abdominal infection in AP. ${ }^{10}$ The body's initial 
response to injury is the local production of inflammatory mediators that lead to the aggregation of inflammatory substances such as white blood cells and platelets and repair of the reticuloendothelial system. ${ }^{11,12}$ However, the microthrombects produced by the aggregation of intravascular platelets contribute to the reduction of platelet destruction. ${ }^{13-15}$ In AP, recent evidence demonstrated that abnormal platelet count was considered as an indicator of coagulation function and severity of the disease. ${ }^{16,17}$

Thrombocytopenia is defined as an abnormal reduction of platelets in the peripheral blood below $10^{\wedge} 10 / \mathrm{L} .{ }^{18}$ Abnormal platelet count is a common complication in patients with AP, and low platelet count often predicts adverse outcomes. ${ }^{19,20}$ As far as we know, coagulation treatment of AP is an important clinical issue. ${ }^{21}$ The decrease in the number of platelets increases the risk of hemorrhage in AP patients. ${ }^{16}$ In addition, the incidence of thrombocytopenia has been strongly associated with ICU mortality in many studies. ${ }^{22-24}$ However, there is still no effective clinical model to predict the occurrence of APassociated thrombocytopenia. Therefore, it is necessary to effectively predict the occurrence of thrombocytopenia or screen out potential risk factors for guiding early clinical intervention to improve prognosis. Machine learning and advances in statistical methodology provide a highefficiency method for the prediction of thrombocytopenia in patients with $\mathrm{AP}$ and can assist clinical investigators and physicians in performing a quick diagnosis especially when there is a high risk of abnormal platelets. Hence, the primary objective of this study was to develop and validate a machine learning model for predicting thrombocytopenia in patients with AP to help clinicians in quick and accurate diagnosis. Further, we hope to design predictive models to monitor coagulation function and guide anticoagulant therapy for AP.

\section{Methods}

\section{Data Sources and Patient Selection}

A retrospective observational study was conducted among a cohort of patients $(\mathrm{n}=594)$ diagnosed with AP. Conducted from May 2018 to May 2019, these data of patients with AP were obtained from the Center of Severe Acute Pancreatitis (CSAP) of Jinling Hospital, Nanjing, China. The center is a tertiary center for AP located in eastern China. All the data were extracted from an electronic database, which stored prospectively collected clinical data of all AP patients admitted to our center. We obtained the approval of the Acute Pancreatitis Database Management Committee (2019NZKY-003-03), and all the analyses were performed in accordance with the committee's regulation. Informed consent involving data storage and academic use of data was obtained from each patient during their hospitalization. The study complies with the Declaration of Helsinki. Patients who met the following criteria were included: (1) diagnosis of AP and (2) time in ICU should be more than 3 days. Patients who were younger than 18 years old, already developed thrombocytopenia before admission and were suspected of neoplasms, aplastic anemia, hypersplenism, and splenomegaly will be excluded from the study.

\section{Definition}

AP (ICD-10, K85) was diagnosed according to the definition in the 2012 revision of the Atlanta classification. ${ }^{3}$ The diagnosis of thrombocytopenia is platelet $<10^{*} 10^{\wedge} 9 / \mathrm{L}$. The patient meeting the diagnosis during the whole hospitalization of AP is calculated into the thrombocytopenia group. Suspected infection was defined as having any culture taken and at least two doses of antimicrobials administered. ${ }^{25}$ To be classified as suspected infection, cultures had to be taken within 24 hours after the start of antimicrobials or antimicrobials had to be started within 72 hours after culture.

\section{Data Collection}

Eighty-nine variables from laboratory examination, vital signs, clinical score, and basic information on the first day after ICU admission, included age, gender, body mass index (BMI), sequential organ failure assessment (SOFA), Glasgow Coma Scale (GCS), acute stage, determinant-based classification type (DBC), infection pancreatic necrosis (IPN), urine output, continuous renal replacement therapy (CRRT), vent, white blood cell (WBC), lymphocyte\% (LYM\%), monocyte\% (MONO\%), red blood cell (RBC), blood urea nitrogen (BUN), creatinine, glucose, globulin, $\mathrm{Ca} 2+$, platelet (PLT), thrombin time (TT), prothrombin time (PT), the international normalised ratio (INR), activated partial thromboplastin time (APTT), D-dimer, fibrinogen (FIB), antithrombase III, red cell distribution width (RDW), myohemoglobin (MB) and so on. All variables are shown in Supplementary Table 1.

\section{Statistical Analysis}

Statistical analyses were performed using SPSS software for Windows Version 25 and R software, version 4.1.1 (R Foundation for Statistical Computing). Continuous 
variables in the data were presented as medians and IQRs or means and SDs. Categorical variables are presented as frequency and percentage. Kolmogorov-Smirnov test was used to test the normality. The student $t$-test or MannWhitney $U$-test and Chi square or Fisher's exact tests were used to investigate the differences in quantitative and categorical variables, respectively between these groups. The LASSO regression method screened the development group's best predictors. Logistic regression was used to build the model with the screened variables and a nomogram was then constructed. The discriminative ability was measured using the area under the ROC curve (AUC). Calibration of the model was assessed by comparison of the predicted and observed probability of thrombocytopenia, accompanied by the HosmerLemeshow goodness-of-fit test. To estimate the clinical usefulness of the nomogram, decision curve analysis (DCA) was built by calculating the net benefits for a range of threshold probabilities. $\mathrm{P}$ value $<0.05$ was considered statistically significant.

\section{Results}

\section{Baseline Patient Characteristics}

The flowchart of the study is shown in Figure 1. There were 1039 patients primarily diagnosed with AP in our ICU from May 2018 to May 2019. 445 patients were excluded for the following reasons: age $<18(n=6)$; incomplete laboratory tests $(n=126)$; ICU days $<3(n=262)$; with cancer $(n=2)$; with thrombocytopenia at the first day after ICU admission $(n=49) .107$ cases were diagnosed as thrombocytopenia (18\%). The training set consisted of 399 patients (thrombocytopenia group 73 cases, nonthrombocytopenia 326 cases), and the validation set was consisted of 195 patients (thrombocytopenia group 34 cases, non-thrombocytopenia 161 cases). Table 1 shows

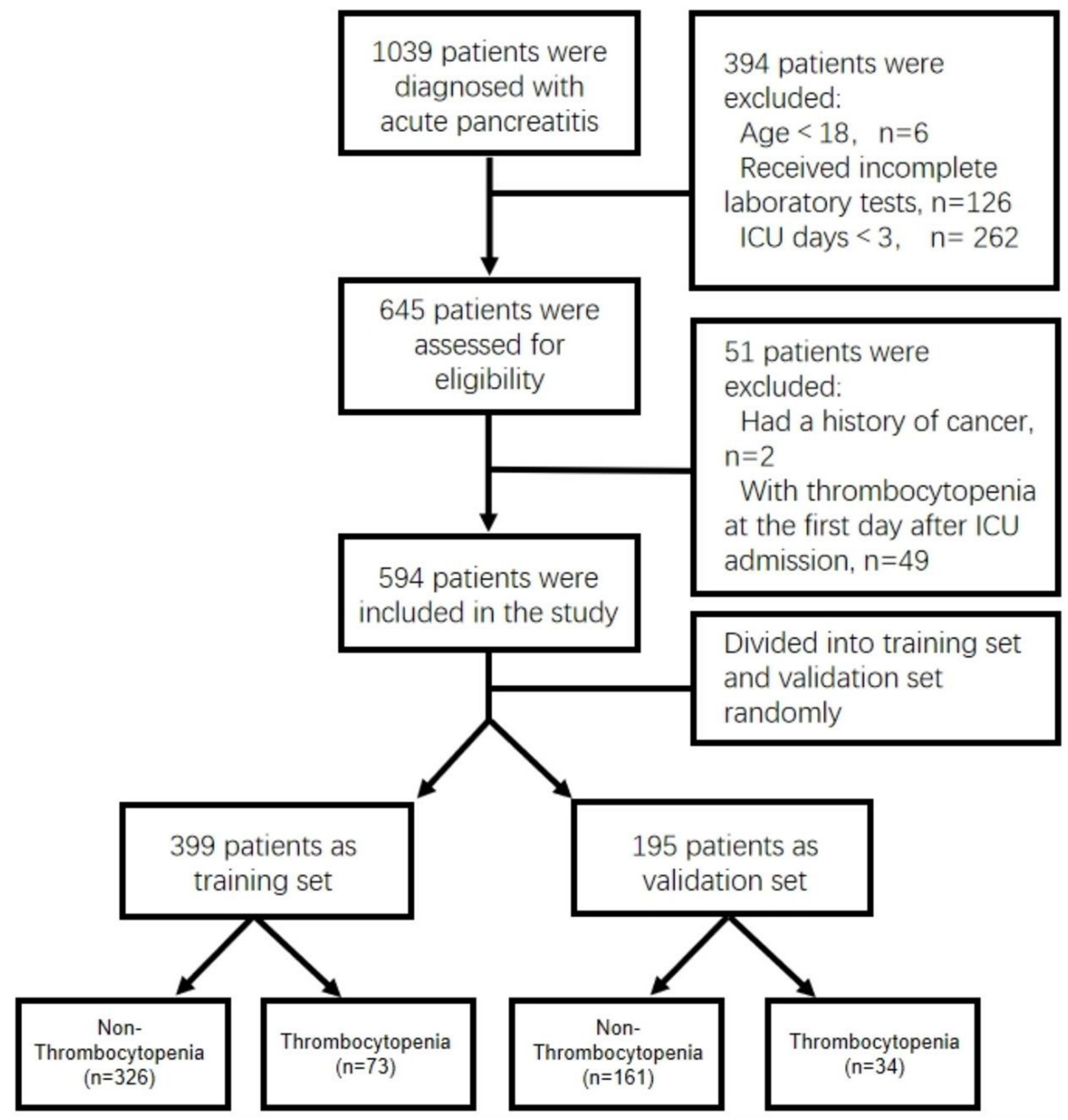

Figure I Flowchart of the study. 
Table I Characteristics of Acute Pancreatitis Patients in Training Set and Validation Set

\begin{tabular}{|c|c|c|c|}
\hline & $\begin{array}{l}\text { Training Set } \\
(n=399)\end{array}$ & $\begin{array}{l}\text { Validation Set } \\
(n=195)\end{array}$ & p value \\
\hline Thrombocytopenia & $73(18.3 \%)$ & $34(17.4 \%)$ & 0.887 \\
\hline Age (year) & $44.8(34.8 \sim 53.5)$ & $42.6(35.0 \sim 53.6)$ & 0.564 \\
\hline Gender & $270(67.7 \%)$ & $126(64.6 \%)$ & 0.517 \\
\hline BMI & $24.6(22.0 \sim 27.5)$ & $24.7(22.0 \sim 27.3)$ & 0.656 \\
\hline SOFA & $3(I \sim 5)$ & $2(0 \sim 5)$ & 0.013 \\
\hline GCS & $15(15 \sim 15)$ & $15(15 \sim 15)$ & 0.697 \\
\hline Acute stage & $137(34.3 \%)$ & $65(15 \%)$ & $0.88 I$ \\
\hline Etiology & & & 0.601 \\
\hline Biliary & $173(16.8 \%)$ & $78(16.8 \%)$ & \\
\hline Hyperlipidemic & $175(16.8 \%)$ & $87(16.8 \%)$ & \\
\hline Others & $51(16.8 \%)$ & $30(16.8 \%)$ & \\
\hline DBC & & & 0.849 \\
\hline Mild & $67(16.8 \%)$ & $35(17.9 \%)$ & \\
\hline Moderate & $162(40.6 \%)$ & $83(42.6 \%)$ & \\
\hline Severe & $118(29.6 \%)$ & $56(28.7 \%)$ & \\
\hline Critical & $52(13.0 \%)$ & $21(10.8 \%)$ & \\
\hline IPN & $87(21.8 \%)$ & $46(23.6 \%)$ & 0.700 \\
\hline Suspected Infection & $187(46.6 \%)$ & $92(47.1 \%)$ & 0.999 \\
\hline Urine output (ml/24h) & $1620(1250 \sim 2340)$ & $1620(1390 \sim 2220)$ & 0.626 \\
\hline CRRT & $31(7.8 \%)$ & $7(3.6 \%)$ & 0.076 \\
\hline Vent & $67(16.8 \%)$ & $26(13.3 \%)$ & 0.333 \\
\hline WBC $\left(\times 10^{9} / L\right)$ & $10(7 \sim 13.8)$ & $10.4(7.1 \sim 14.4)$ & 0.705 \\
\hline Ly $\left(\times 10^{9} / L\right)$ & $10.1(6.8 \sim 15.8)$ & $10.2(7 \sim 14.9)$ & 0.912 \\
\hline MONO $\left(\times 10^{9} / \mathrm{L}\right)$ & $6.3(4.6 \sim 8.4)$ & $6.2(4.5 \sim 8.5)$ & 0.938 \\
\hline $\operatorname{RBC}\left(\times 10^{12} / \mathrm{L}\right)$ & $3.3(2.8 \sim 3.8)$ & $3.4(2.9 \sim 3.9)$ & 0.193 \\
\hline BUN (mmol/L) & $4.7(3.2 \sim 7.9)$ & $4.7(3.3 \sim 7.2)$ & 0.657 \\
\hline Creatinine $(\mu \mathrm{mol} / \mathrm{L})$ & $56.5(42.1 \sim 79)$ & $52.3(40.3 \sim 72.3)$ & 0.125 \\
\hline Glucose (mmol/L) & $7.2(5 \sim 10)$ & $7.4(5.4 \sim 10.2)$ & 0.377 \\
\hline Globulin (g/L) & $26.6(23 \sim 29.8)$ & $26.9(22 \sim 31.2)$ & 0.749 \\
\hline $\mathrm{Ca}^{2+}(\mathrm{mmol} / \mathrm{L})$ & $2.1(2.0 \sim 2.2)$ & $2.1(2.0 \sim 2.2)$ & 0.950 \\
\hline PLT $\left(\times 10^{9} / \mathrm{L}\right)$ & $206(160 \sim 293)$ & $219(|7| \sim 302)$ & 0.107 \\
\hline TT (s) & $16.6(15.3 \sim 18.8)$ & $16.5(15.1 \sim 18.2)$ & 0.410 \\
\hline PT (s) & $13.8(13 \sim 15)$ & |3.8(|2.8 |5.4) & 0.583 \\
\hline INR & $1.2(1.1 \sim 1.3)$ & $1.2(1.1 \sim 1.3)$ & 0.396 \\
\hline APTT (s) & $3 \mathrm{I} . \mathrm{I}(27.5 \sim 36.1)$ & $30.5(27.3 \sim 35.2)$ & 0.397 \\
\hline
\end{tabular}

(Continued) 
Table I (Continued).

\begin{tabular}{|c|c|c|c|}
\hline & $\begin{array}{l}\text { Training Set } \\
(n=399)\end{array}$ & $\begin{array}{l}\text { Validation Set } \\
(n=195)\end{array}$ & $p$ value \\
\hline D-dimer $(\mu \mathrm{g} / \mathrm{L})$ & $4.6(2.2 \sim 8.6)$ & $4.0(2.3 \sim 7.3)$ & 0.434 \\
\hline $\mathrm{FIB}(g / \mathrm{L})$ & $4.3(3.2 \sim 5.4)$ & $4.4(3.4 \sim 5.5)$ & 0.325 \\
\hline Antithrombase III (\%) & $66.5(55 \sim 79)$ & $67.4(55.6 \sim 78.4)$ & 0.815 \\
\hline RDW (\%) & |4. $\mid(\mid 3.2 \sim 15.2)$ & $|4|.(|3.| \sim 15.1)$ & 0.314 \\
\hline $\mathrm{MB}(\mathrm{ng} / \mathrm{mL})$ & $36.9(21 \sim 95.7)$ & $36.2(20 \sim 83.8)$ & 0.523 \\
\hline
\end{tabular}

the main baseline characteristics of patients in the training set and validation set. The thrombocytopenia incidence was comparable between two data sets $(18.3 \%$ vs $17.4 \%$; $\mathrm{P}=0.887$ ). The SOFA of patients was higher in the training set. There were no significant differences in age, gender, BMI, or other comorbidities between these two data sets.

Patients in the thrombocytopenia group were older than those in the non-thrombocytopenia group $(\mathrm{P}<0.001)$. Significant differences were observed in the clinic scores and laboratory indicators between these two groups. In summary, patients in the thrombocytopenia group had more severe organ function damage, a hypercoagulable state, and increased renal injury compared with those in the non-thrombocytopenia group (Table 2).

\section{Nomogram Development}

The association of the variables with thrombocytopenia is presented in Supplementary Table 1 . There were 5 parameters selected by the LASSO-penalized logistic regression model, including BUN, FIB, MB, antithrombase III and BNPP (Figure 2A and B). Multivariate logistic analysis showed three independent risk-predictive factors for thrombocytopenia: BUN, FIB, antithrombase III (Table 3). A nomogram was established to determine the influence of the three variables on thrombocytopenia (Figure 3). Antithrombase III showed the most extended scale and the most significant effect on thrombocytopenia.

The nomogram and dynamic nomogram applications are described as follows: The scores corresponding to each variable can be obtained, and the sum of these scores is recorded as the total score. The prediction risk corresponding to the total score is the risk of thrombocytopenia in AP (Figure 3). To obtain the dynamic nomogram, visit https:// hongbindeng.shinyapps.io/nomogram/. It is a practical and accurate online tool (Supplementary Figure 1).

\section{Evaluation and Validation of the Dynamic Nomogram Discrimination and Calibration}

The c-statistic of the nomogram was 0.881 in the training set data (Figure 4A), and 0.883 in the validation set (Figure 4B). The $\mathrm{P}$ value for the Hosmer-Lemeshow test was both more than 0.05 in the training set and validation set $(\mathrm{P}=0.339, \mathrm{P}=0.464$; respectively). The calibration curves are shown in Figure $4 \mathrm{C}$, and D. The Brier scores in the development and validation groups were 0.096 and 0.098, respectively. Both the Hosmer-Lemeshow tests and the calibration curves showed that the model's predicted probability was highly consistent with the actual probability, and the model had good calibrations in both the training and validation sets.

\section{Decision Curve Analysis}

Decision curve analysis results of the risk nomogram in the training set and validation set were shown to determine an optimal decision point of the nomogram (Figure $4 \mathrm{E}$ and F). For predicted risk thresholds between $0 \%$ and $63 \%$, the nomogram model showed a positive net benefit in two data sets.

\section{Discussion}

The changes of coagulation function during AP greatly affect the clinical manifestations and prognosis of patients. However, the question of whether platelets are directly involved in the pathophysiology of AP remains uncertain. $^{26-28}$ It is clear that in the early stages of AP, the leucocyte - endothelial cell response in the pancreatic microcirculation causes vascular damage that recruits platelet activation. ${ }^{15,29}$ Upon activation, platelets can generate reactive oxygen species and nitric oxide as well as release pro-inflammatory mediators. Furthermore, the accumulation 
Table 2 Characteristics of Acute Pancreatitis Patients in Thrombocytopenia Set and Non-Thrombocytopenia Set

\begin{tabular}{|c|c|c|c|}
\hline & $\begin{array}{l}\text { Thrombocytopenia } \\
(n=107)\end{array}$ & $\begin{array}{l}\text { Non-Thrombocytopenia } \\
(n=487)\end{array}$ & p value \\
\hline Age (year) & $50.6(38.0 \sim 60.8)$ & $43.9(34.0 \sim 51.4)$ & $<0.001$ \\
\hline Gender & $69(64.4 \%)$ & $327(67.1 \%)$ & 0.678 \\
\hline BMI & $24.2(22.3 \sim 26.9)$ & $24.7(22.0 \sim 27.6)$ & 0.535 \\
\hline SOFA & $6(3 \sim 8)$ & $2(0 \sim 4)$ & $<0.001$ \\
\hline GCS & $15(15 \sim 15)$ & $15(15 \sim 15)$ & $<0.001$ \\
\hline Acute stage & $16(14.9 \%)$ & $186(38.1 \%)$ & $<0.001$ \\
\hline Etiology & & & 0.012 \\
\hline Biliary & $58(54.2 \%)$ & $193(39.6 \%)$ & \\
\hline Hyperlipidemic & $34(31.7 \%)$ & $228(46.8 \%)$ & \\
\hline Others & $15(14.0 \%)$ & $66(13.5 \%)$ & \\
\hline $\mathrm{DBC}$ & & & $<0.001$ \\
\hline Mild & $7(6.5 \%)$ & $95(19.5 \%)$ & \\
\hline Moderate & $27(25.2 \%)$ & $218(44.7 \%)$ & \\
\hline Severe & $44(41.1 \%)$ & $130(26.6 \%)$ & \\
\hline Critical & $29(27.1 \%)$ & $44(9.0 \%)$ & \\
\hline IPN & $36(33.6 \%)$ & $97(19.9 \%)$ & 0.003 \\
\hline Suspected Infection & $57(53.2 \%)$ & $222(45.5 \%)$ & 0.182 \\
\hline Urine output (ml/24h) & $1540(907.5 \sim 1820)$ & $1620(1450 \sim 2350)$ & $<0.001$ \\
\hline CRRT & $14(13.0 \%)$ & $24(4.9 \%)$ & 0.004 \\
\hline Vent & $43(40.1 \%)$ & $50(10.2 \%)$ & $<0.001$ \\
\hline WBC $\left(\times 10^{9} / L\right)$ & $10.4(7.3 \sim 14.8)$ & $10.1(7.0 \sim 13.8)$ & 0.358 \\
\hline Ly $\left(\times 10^{9} / \mathrm{L}\right)$ & $0.9(0.5 \sim 1.4)$ & $1.0(0.7 \sim 1.6)$ & 0.088 \\
\hline MONO $\left(\times 10^{9} / \mathrm{L}\right)$ & $4.8(3.5 \sim 7.0)$ & $6.5(4.8 \sim 8.7)$ & $<0.001$ \\
\hline $\operatorname{RBC}\left(\times 10^{12} / \mathrm{L}\right)$ & $2.8(2.5 \sim 3.2)$ & $3.5(2.9 \sim 4.0)$ & $<0.001$ \\
\hline BUN (mmol/L) & $9.7(4.9 \sim 17.0)$ & $4.3(3.1 \sim 3.2)$ & $<0.001$ \\
\hline Creatinine $(\mu \mathrm{mol} / \mathrm{L})$ & $82.7(51.9 \sim 201.3)$ & $52.6(40.9 \sim 68.9)$ & $<0.001$ \\
\hline Glucose (mmol/L) & $8.7(6.5 \sim 13.3)$ & $6.8(5.1 \sim 9.5)$ & $<0.001$ \\
\hline Globulin (g/L) & $27.5(23.1 \sim 32.1)$ & $26.6(22.6 \sim 30.1)$ & 0.206 \\
\hline $\mathrm{Ca}^{2+}(\mathrm{mmol} / \mathrm{L})$ & $2.1(1.9 \sim 2.2)$ & $2.1(2.0 \sim 2.2)$ & 0.035 \\
\hline PLT $\left(\times 10^{9} / \mathrm{L}\right)$ & $\mid 87(|4| \sim 287)$ & $213(169 \sim 300)$ & 0.006 \\
\hline TT (s) & $17.1(15.9 \sim 19.7)$ & $16.4(\mid 5.1 \sim 18.1)$ & 0.001 \\
\hline PT (s) & $15.3(\mid 3.8 \sim 17.0)$ & $13.7(\mid 2.8 \sim 14.6)$ & $<0.001$ \\
\hline INR & $1.3(1.2 \sim 1.5)$ & $1.2(1.1 \sim 1.3)$ & $<0.001$ \\
\hline APTT (s) & $34.2(29.1 \sim 40.6)$ & $30.3(27.1 \sim 34.8)$ & $<0.001$ \\
\hline D-dimer $(\mu g / L)$ & $6.5(3.6 \sim 9.2)$ & $4.2(2.1 \sim 7.6)$ & $<0.001$ \\
\hline
\end{tabular}

(Continued) 
Table 2 (Continued).

\begin{tabular}{|l|l|l|l|}
\hline & $\begin{array}{l}\text { Thrombocytopenia } \\
(\mathbf{n}=107)\end{array}$ & $\begin{array}{l}\text { Non-Thrombocytopenia } \\
(\mathbf{n}=487)\end{array}$ & v value \\
\hline FIB (g/L) & $3.4(2.7 \sim 4.4)$ & $4.5(3.4 \sim 5.7)$ & $<0.00 \mathrm{I}$ \\
\hline Antithrombase III (\%) & $51.5(40.8 \sim 63.8)$ & $69.9(59.2 \sim 81.3)$ & $<0.001$ \\
\hline RDW (\%) & $15.1(14.2 \sim 16.3)$ & $13.8(13.0 \sim 14.9)$ & $<0.001$ \\
\hline MB $(\mathrm{ng} / \mathrm{mL})$ & $116.8(34.8 \sim 531.2)$ & $33.0(20.0 \sim 66.7)$ & $<0.001$ \\
\hline
\end{tabular}

of platelets in the microvasculature during the reperfusion period enhances the recruitment of activated leukocytes, thereby exacerbating the intensity of the inflammatory response. ${ }^{30,31}$ Therefore, from this point of view platelets can exacerbate the injury of AP while consuming themselves in a vicious cycle and even inducing thrombocytopenia.

In our study, we developed and validated a machine learning-based model that incorporates the clinical changes of patients and the platelet signature and distribution, individualized prediction of thrombocytopenia in patients with AP. The nomogram demonstrated favorable discrimination in both the training set (AUC, 0.881) and the validation set cohort (AUC, 0.883) and good calibration, which confirms that the training-validation model achieved excellent predictive efficacy. Decision curve analysis (DCA) indicated the clinical usefulness of the mixed model. Moreover, our prediction model is straightforward and can be used directly with online tools to avoid the computational errors associated with standard nomogram. From this point of view, clinicians can actively carry out early fluid resuscitation and close monitoring according to the early predictive value of patients with AP, and even timely transfer patients to pancreatic hospitals or large medical institutions with better conditions.

In this study, almost all clinical data of the patients in our AP center were included and the machine learning model was used for step-by-step double-validation. Finally, laboratory blood indicators BUN, FIB, and antithrombase III were screened for the first time and confirmed to be able to predict the occurrence of thrombocytopenia after AP efficiently and accurately. To our knowledge, blood urea nitrogen (BUN) is a well-established marker of mortality in AP, which may explain its potential
A

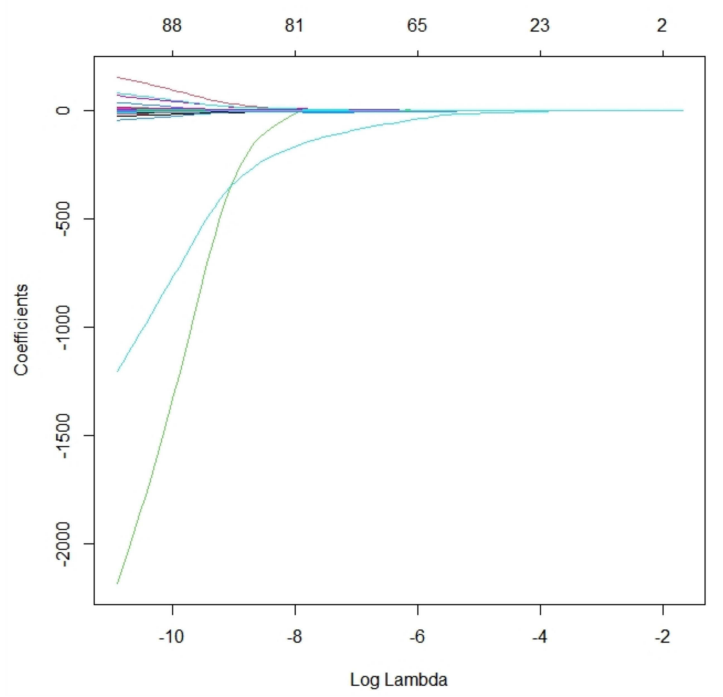

B

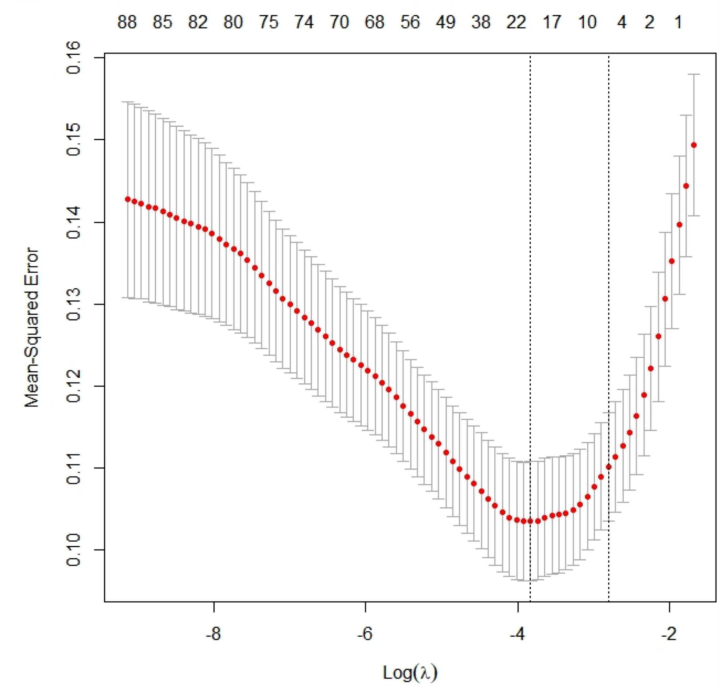

Figure 2 Identification of significant predictors for the thrombocytopenia in patients with acute pancreatitis. (A) LASSO coefficient profiles of the candidate predictors. (B) Selection of the optimal penalization coefficient in the LASSO regression. 
Table 3 The Result of Multivariate Analysis

\begin{tabular}{|c|c|c|c|c|c|c|}
\hline \multirow[t]{2}{*}{ Variables } & Training Set & & Validation Set & & Total & \\
\hline & OR $(95 \% \mathrm{Cl})$ & p value & OR $(95 \% \mathrm{Cl})$ & p value & OR $(95 \% \mathrm{Cl})$ & p value \\
\hline BUN (mmol/L) & I. $160(1.098 \sim 1.225)$ & $<0.001$ & I.I95 (I.077 1.325) & 0.001 & $1.165(1.111 \sim 1.221)$ & $<0.001$ \\
\hline FIB $(g / L)$ & $0.694(0.539 \sim 0.893)$ & 0.005 & $0.609(0.433 \sim 0.856)$ & 0.004 & $0.668(0.546 \sim 0.817)$ & $<0.001$ \\
\hline $\mathrm{MB}(\mathrm{ng} / \mathrm{mL})$ & $1.000(1.000 \sim 1.001)$ & 0.128 & $1.000(1.000 \sim 1.001)$ & 0.083 & $1.000(1.000 \sim 1.001)$ & 0.022 \\
\hline Antithrombase III (\%) & $0.946(0.924 \sim 0.967)$ & $<0.001$ & $0.948(0.9 \mid 8 \sim 0.978)$ & 0.001 & $0.947(0.930 \sim 0.964)$ & $<0.001$ \\
\hline BNPP (pmol/L) & $1.002(1.000 \sim 1.005)$ & 0.034 & $0.999(0.996 \sim 1.002)$ & 0.472 & $1.001(0.999 \sim 1.003)$ & 0.242 \\
\hline
\end{tabular}

predictive value in AP-associated thrombocytopenia. ${ }^{32,33}$ In addition, antithrombase III reduction is associated with severity of AP, low levels could be caused by consumption due to coagulopathy or increased elimination due to renal complications. ${ }^{34}$ Both are indicative for AP severity and can cause thrombocytopenia. ${ }^{35}$ Fibrinogen (FIB) is an acute-phase protein with commonly high plasma levels due to the inflammatory state of AP. ${ }^{36}$ When FIB is low, it is again a sign of consumption (eg in DIC) secondary to AP causing thrombocytopenia. ${ }^{37}$ As for the effects of disseminated intravascular coagulation (DIC), although some patients met the criteria based on ISTH Diagnostic Scoring System for DIC, none of the patients included in the analysis was diagnosed with DIC during their ICU stay (Supplementary Table 2). This may mean that the DIC score is not appropriate for AP, and a special DIC score for AP should be developed. In other words, although DIC constitutes a potential cause of thrombocytopenia, it is not present in our patients. This suggests that we should pay attention to DIC problems in the diagnosis and treatment of AP in the future to avoid missed diagnosis and misdiagnosis. Finally, it is generally known that AP can lead

Points

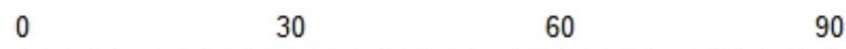

BUN

Fibrinogen

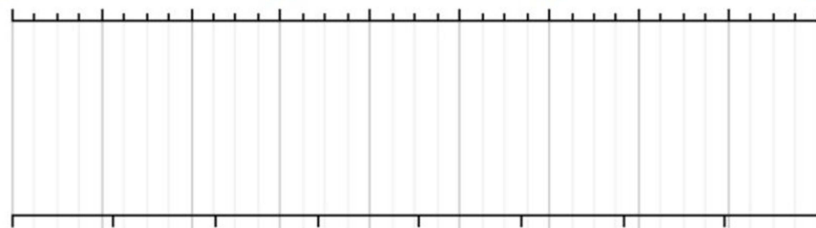

$$
0
$$

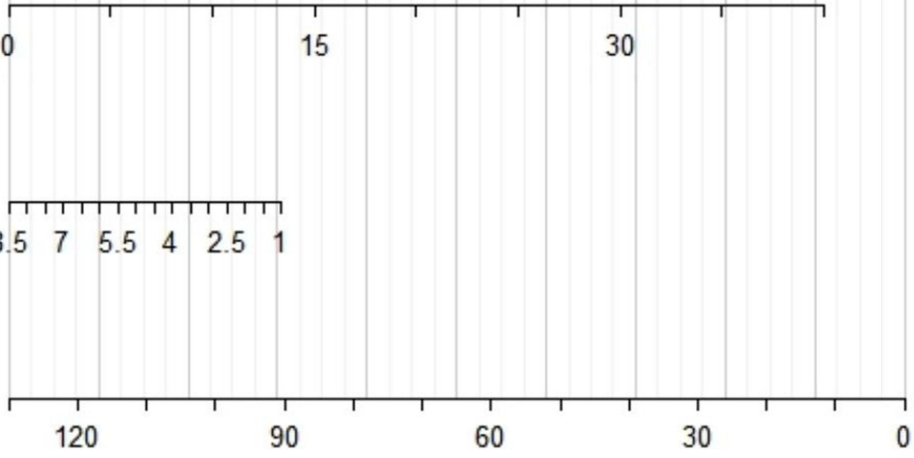

Total Points

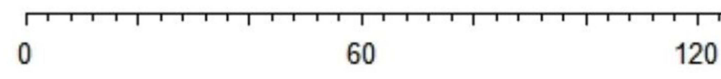

Risk of thrombocytopenia

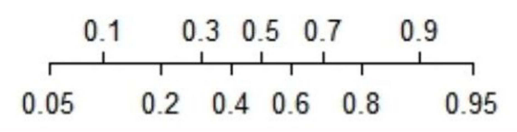

Figure 3 Construction and validation of the predictive nomogram for the thrombocytopenia in patients with acute pancreatitis. 
A

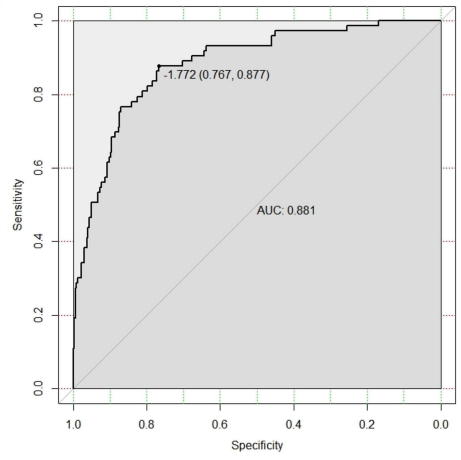

B

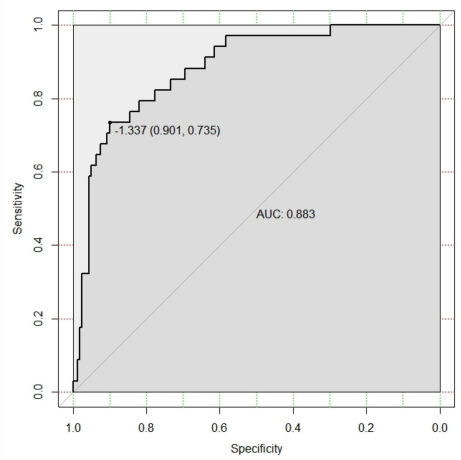

C

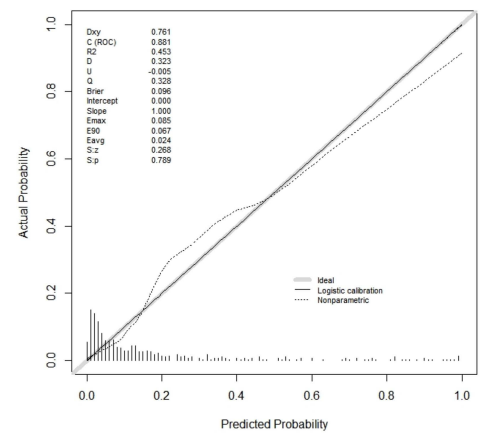

D

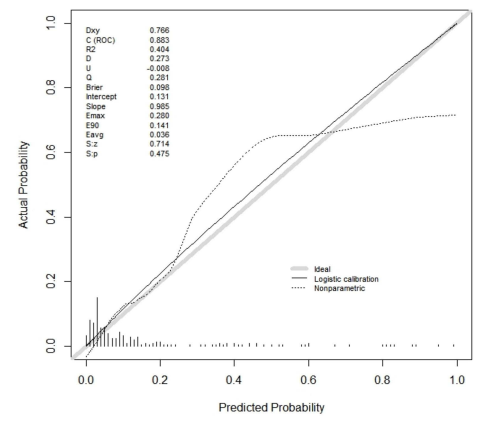

E

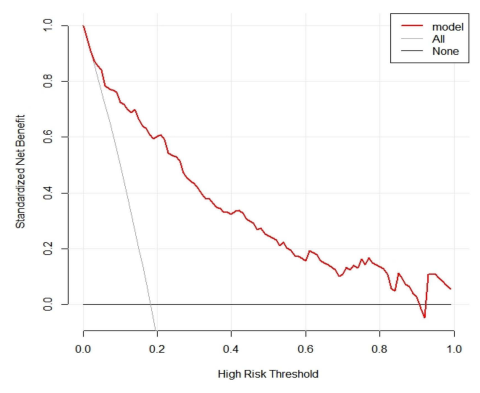

F

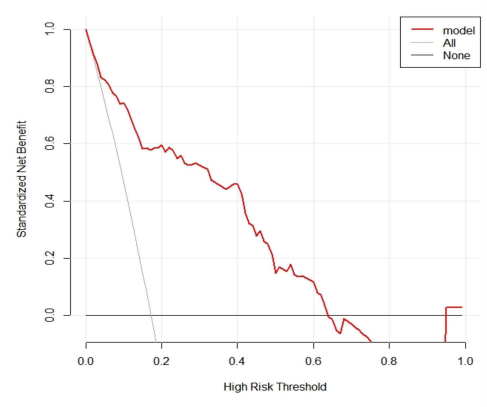

Figure 4 (A) Receiver operating characteristic (ROC) curves of the nomogram in the training cohort. (B) ROC curves of the nomogram in the validation cohort. (C) Calibration curve of the nomogram in the training cohort. (D) Calibration curve of the nomogram in the validation cohort. (E) Decision curve analysis in the training cohort. (F) Decision curve analysis in the validation cohort.

to thrombotic thrombocytopenic purpura (TTP), yet this cause was also not present in our patient.

Large amounts of platelets are activated and consumed in the early stages of AP, and the mean platelet volume (MPV) is considered to be a marker of platelet depletion and the resulting compensatory bone marrow response over the course of the disease. In other words, elevated mean platelet volume is a marker of organ failure and platelet depletion in patients with AP. Furthermore, thrombocytopenia caused by persistent platelet depletion is considered to be an adverse complication of SAP, which is consistent with recent reports that platelet levels predict the severity of AP. ${ }^{16,17}$ In our study, our nomogram performed well in both the training set and the validation set, and it also showed good calibration in both the training and validation samples.

In order to go beyond pure mathematics and evaluate the model from the perspective provided by performance measures such as AUC, DCA is used to evaluate the expected net benefit of the model under all possible risk thresholds, thereby assessing the impact of various risk thresholds. ${ }^{38,39}$ DCA showed that if the threshold probability was between
$0 \%$ and $63 \%$, using the nomogram in the current study to predict thrombocytopenia in patients with AP adds more benefit than either of these two data sets. Therefore, our research mainly includes technological innovations and advantages in the following aspects: firstly, this is the first predictive model developed for patients with thrombocytopenia in AP; secondly, the results are displayed in dynamic nomogram, and the data can be input directly, which avoids the calculation difficulty of continuous variables and improves the validity of the model; thirdly, DCA was introduced in our study to make risk stratification more intuitive and reasonable.

However, there are some limitations to our analysis. The major limitation of the present study might be that it was a single-centre retrospective in nature, and selection bias cannot be ignored. Secondly, since we collected platelet counts from hospitalized patients in the acute phase, a small number of patients were diagnosed with thrombocytopenia for some transient reason, thus potentially causing bias. Thirdly, the number of patients included in the analysis in this study is still limited, so further validation with a large 
sample is necessary. Finally, there are some clinical questions of thrombocytopenia in patients with AP not to be resolved, such as classification of severity and exploration of the clinical relationship. In the next step, we will continue to carry out multidisciplinary cooperation, and select more precise features to develop a deep learning model, and also should focus on multicenter validation with a larger sample size to obtain high-level evidence for the clinical application of deep learning model. In this way, clinicians and patients can benefit to a greater extent.

In conclusion, the nomogram model, which is consisted of three independent risk factors for thrombocytopenia, may empower clinicians with earlier and more accurate information regarding the risk of thrombocytopenia and help in clinical decision-making.

\section{Data Sharing Statement}

The datasets used or analysed during the current study are available from the corresponding author (Weiqin $\mathrm{Li}$ ) on reasonable request.

\section{Consent for Publication}

All authors have agreed to publish this article.

\section{Author Contributions}

All authors made a significant contribution to the work reported, whether that is in the conception, study design, execution, acquisition of data, analysis and interpretation, or in all these areas; took part in drafting, revising, or critically reviewing the article; gave final approval of the version to be published; have agreed on the journal to which the article has been submitted; and agree to be accountable for all aspects of the work.

\section{Funding}

The study was supported by the National Natural Science Foundation of China (82070669 to W.L.). The sponsor had no role in the design of the study, the collection and analysis of the data, or the preparation of the manuscript.

\section{Disclosure}

The authors report no conflicts of interest in this work.

\section{References}

1. Thandassery RB, Yadav TD, Dutta U, et al. Dynamic nature of organ failure in severe acute pancreatitis: the impact of persistent and deteriorating organ failure. $H P B$. 2013;15(7):523-528. doi:10.1111/j.14772574.2012.00613.x
2. Salomone T, Tosi P, Palareti G, et al. Coagulative disorders in human acute pancreatitis: role for the D-dimer. Pancreas. 2003;26 (2):111-116. doi:10.1097/00006676-200303000-00003

3. Banks PA, Bollen TL, Dervenis C, et al.; Acute Pancreatitis Classification Working. Classification of acute pancreatitis-2012: revision of the Atlanta classification and definitions by international consensus. Gut. 2013;62(1):102-111. doi:10.1136/gutjnl-2012-302 779

4. Windsor JA, Escott A, Brown L, et al. Novel strategies for the treatment of acute pancreatitis based on the determinants of severity. J Gastroenterol Hepatol. 2017;32(11):1796-1803. doi:10. 1111/jgh. 13784

5. Dominguez-Munoz JE, Viedma JA, Perez-Mateo $M$, et al. Inflammatory response in the initial phase of acute pancreatitis: relationship to the onset and severity of the disease. Rev Esp Enferm Dig. 1995;87:225Y246.

6. Bhatia M. Novel therapeutic targets for acute pancreatitis and associated multiple organ dysfunction syndrome. Curr Drug Targets Inflamm Allergy. 2002;1(4):343Y351. doi:10.2174/156801002334 4517

7. Rinderknecht H. Fatal pancreatitis, a consequence of excessive leukocyte stimulation? Int J Pancreatol. 1988;3(2-3):105Y112. doi:10. 1007/BF02798921

8. Bhatia M, Saluja AK, Hofbauer B, et al. Neutral endopeptidase (NEP) plays an anti-inflammatory role in acute pancreatitis and pancreatitis-associated lung injury. Pancreas. 1997;15:428.

9. Algul H, Tando Y, Schneider G, et al. Acute experimental pancreatitis and NF-kappaB/Rel activation. Pancreatology. 2002;2(6):503Y509. doi:10.1159/000066090

10. Nijsten MW, Ten Duis HJ, Zijlstra JG, et al. Blunted rise in platelet count in critically ill patients is associated with worse outcome. Crit Care Med. 2000;28(6):1871. doi:10.1097/00003246-20001200000017

11. Stephan F, Hollande J, Richard O, et al. Thrombocytopenia in a surgical ICU. Chest. 1999;115(5):1363. doi:10.1378/chest.115.5. 1363

12. Scherer RU, Spangenberg P. Procoagulant activity in patients with isolated head trauma. Crit Care Med. 1998;26(1):149. doi:10.1097/ 00003246-199801000-00031

13. von Dobschuetz E, Bleiziffer O, Pahernik S, et al. Soluble complement receptor 1 preserves endothelial barrier function and microcirculation in postischemic pancreatitis in the rat. Am $J$ Physiol Gastrointest Liver Physiol. 2004;286(5):G791-G796. doi:10.1152/ ajpgi.00407.2003

14. Mayer H, Schmidt J, Tbies J, et al. Characterization and reduction of ischemia/reperfusion injury after experimental pancreas transplantation. J Gastrointest Surg. 1999;3(2):162-166. doi:10.10 16/S1091-255X(99)80027-4

15. Mimidis K, Papadopoulos V, Kotsianidis J, et al. Alterations of platelet function, number and indexes during acute pancreatitis. Pancreatology. 2004;4(1):22-27. doi:10.1159/000077024

16. Huang P, Zhang Y, Wu H. Mean platelet volume as an indicator of persistent organ failure in acute pancreatitis. Int J Clin Exp Pathol. 2016;9:12883-12889.

17. Papadopoulos VP, Filippou DK, Mimidis KP, et al. Mean platelet volume in acute pancreatitis: a systematic review and meta-analysis. Ann Gastroenterol. 2020;33(4):398-404. doi:10.20524/aog.2020.0495

18. van den Hurk K, Veenstra J, Hendriks HA, et al. [Thrombocytopenia]. Ned Tijdschr Geneeskd. 2016;160:D417. Dutch

19. Gasparyan AY, Ayvazyan L, Mikhailidis DP, Kitas GD. Mean platelet volume: a link between thrombosis and inflammation? Curr Pharm Des. 2011;17(1):47-58. doi:10.2174/138161211795049804

20. Erbis H, Aliosmanoglu I, Turkoglu MA, Ay E, Turkoglu A, Ulger BV. Evaluating mean platelet volume as a new indicator for confirming the diagnosis of necrotizing pancreatitis. Ann Ital Chir. 2014;86:132-136. 
21. Tomkotter L, Erbes J, Trepte C, et al. The effects of pancreatic microcirculatory disturbances on histopathologic tissue damage and the outcome in severe acute pancreatitis. Pancreas. 2016;45 (2):248-253. doi:10.1097/MPA.0000000000000440

22. Guru PK, Singh TD, Akhoundi A, et al. Association of thrombocytopenia and mortality in critically ill patients on continuous renal replacement therapy. Nephron. 2016;133(3):175-182. doi:10.1159/000447543

23. Menard CE, Kumar A, Houston DS, et al. Evolution and impact of thrombocytopenia in septic shock: a retrospective cohort study. Crit Care Med. 2019;47(4):558-565. doi:10.1097/CCM.0000000000003644

24. Burunsuzoglu B, Saltürk C, Karakurt Z, et al. Thrombocytopenia: a risk factor of mortality for patients with sepsis in the intensive care unit. Turk Thorac J. 2016;17(1):7-14. doi:10.5578/ttj.17.1.002

25. Valik JK, Mellhammar L, Sundén-Cullberg J, et al. Peripheral oxygen saturation facilitates assessment of respiratory dysfunction in the sequential organ failure assessment score with implications for the sepsis-3 criteria. Crit Care Med. 2021. doi:10.1097/CCM.000 0000000005318

26. Uhlmann D, Lauer H, Serr F, et al. Pathophysiological role of platelets and platelet system in acute pancreatitis. Microvasc Res. 2008;76 (2):114-123. doi:10.1016/j.mvr.2008.05.001

27. Kakafika A, Papadopoulos V, Mimidis K, Mikhailidis DP. Coagulation, platelets, and acute pancreatitis. Pancreas. 2007;34 (1):15-20. doi:10.1097/01.mpa.0000240617.66215.d2

28. Witzigmann H, Ludwig S, Armann B, et al. Endothelin (A) receptor blockade reduces ischemia/reperfusion injury in pig pancreas transplantation. Ann Surg. 2003;238(2):264-274. doi:10.1097/01. sla.0000080830.77437.4f

29. Osada J, Wereszczynska-Siemiatkowska U, Dabrowski A, Dabrowska MI. Platelet activation in acute pancreatitis. Pancreas. 2012;41(8):1319-1324. doi:10.1097/MPA.0b013e31824bd89f

30. Zaninovic V, Gukovskaya AS, Gukovsky I, et al. Cerulein upregulates ICAM-1 in pancreatic acinar cells, which mediates neutrophil adhesion to these cells. Am J Physiol Gastrointest Liver Physiol. 2000;279(4):G666-G676. doi:10.1152/ajpgi.2000.279.4.G666
31. Frossard JL, Saluja A, Bhagat L, et al. The role of intercellular adhesion molecule 1 and neutrophils in acute pancreatitis and pancreatitis-associated lung injury. Gastroenterology. 1999;116 (3):694-701. doi:10.1016/S0016-5085(99)70192-7

32. Lin S, Hong W, Basharat Z, et al. Blood urea nitrogen as a predictor of severe acute pancreatitis based on the revised Atlanta criteria: timing of measurement and cutoff points. Can J Gastroenterol Hepatol. 2017;2017:9592831. doi:10.1155/2017/9592831

33. Wu BU, Johannes RS, Sun X, et al. Early changes in blood urea nitrogen predict mortality in acute pancreatitis. Gastroenterology. 2009;137(1):129-135. doi:10.1053/j.gastro.2009.03.056

34. Kroner PT, Wallace MB, Raimondo M, et al. Systemic anticoagulation is associated with decreased mortality and morbidity in acute pancreatitis. Pancreatology. 2021;21(8):1428-1433. doi:10.1016/j. pan.2021.09.003

35. Yang N, Hao J, Zhang D. Antithrombin III and D-dimer levels as indicators of disease severity in patients with hyperlipidaemic or biliary acute pancreatitis. J Int Med Res. 2017;45(1):147-158. doi:10.1177/0300060516677929

36. Liu C, Zhou X, Ling L, et al. Prediction of mortality and organ failure based on coagulation and fibrinolysis markers in patients with acute pancreatitis: a retrospective study. Medicine. 2019;98(21):e15648. doi:10.1097/MD.0000000000015648

37. $\mathrm{Xu} \mathrm{M}$, Luo L, Du M, et al. Fibrinogen levels are associated with bleeding in patients with primary immune thrombocytopenia. Platelets. 2020;31(6):763-770. doi:10.1080/09537104.2019.1678115

38. Collins GS, Reitsma JB, Altman DG, Moons KG. Transparent reporting of a multivariable prediction model for individual prognosis or diagnosis (TRIPOD): the TRIPOD statement. BJOG. 2015;122 (3):434 443. doi:10.1111/1471-0528.13244

39. Vickers AJ, Elkin EB. Decision curve analysis: a novel method for evaluating prediction models. Med Decis Making. 2006;26 (6):565-574. doi:10.1177/0272989X06295361
Journal of Inflammation Research

\section{Publish your work in this journal}

The Journal of Inflammation Research is an international, peerreviewed open-access journal that welcomes laboratory and clinical findings on the molecular basis, cell biology and pharmacology of inflammation including original research, reviews, symposium reports, hypothesis formation and commentaries on: acute/chronic inflammation; mediators of inflammation; cellular processes; molecula mechanisms; pharmacology and novel anti-inflammatory drugs; clinical conditions involving inflammation. The manuscript management system is completely online and includes a very quick and fair peerreview system. Visit http://www.dovepress.com/testimonials.php to read real quotes from published authors. 